\title{
La combinación de finasteride y doxazosina fue más efectiva que la monoterapia en la hiperplasia prostática benigna
}

\section{Objetivo:}

Evaluar la eficacia de doxazosina, finasteride y la combinación de ambas en la progresión de la hiperplasia prostática benigna (HPB) a largo plazo.

\section{Diseño:}

Ensayo clínico controlado aleatorizado, doble ciego, a 4,5 años de seguimiento promedio.

Lugar:

Se llevó a cabo en 17 centros de los EE.UU.

\section{Pacientes:}

Un total de 3047 pacientes de edad promedio de 63 años, con síntomas moderados a severos según el Índice Sintomático de la Asociación Urológica Americana (ISAU, ver anexo en página adjunta) y con antígeno prostático específico (APE) $<10 \mathrm{ng} / \mathrm{ml}$.

\section{Intervención}

Los pacientes fueron divididos en 4 ramas: placebo, finasteride 5 $\mathrm{mg}$, doxazosina hasta $8 \mathrm{mg}$ (según tolerancia) y la combinación de estas dos últimas.

\section{Medición de Resultados Principales:}

Se definió la progresión de los síntomas mediante alguno de los siguientes: Incremento del ISAU de al menos 4 puntos (cambio clínicamente significativo), retención urinaria, infección urinaria, incontinencia urinaria e insuficiencia renal. Fueron evaluadas también la necesidad de procedimiento quirúrgico, la modificación del flujo urinario, el APE y volumen prostático.

\section{Resultados:}

Ocurrieron 351 eventos: 128 en el grupo placebo, 85 en el grupo doxazosina, 89 en el de finasteride y 49 en el grupo combinado.

El $78 \%$ de los eventos fueron un incremento de 4 puntos en la escala ISAU, un $12 \%$ fueron ITUs, $9 \%$ incontinencia, no se reportaron casos de insuficiencia renal. En cuanto a la progresión clínica de más de cuatro puntos de la escala, la tasa de progresión cada 100 personas/año es de 4,5 para el grupo placebo, 2,7 para el grupo doxazosina, 2,9 para los tratados con finasteride y 1,5 para el tratamiento combinado. Comparados con el placebo, la reducción de la progresión de enfermedad al año fue significativamente mayor en el grupo de tratamiento combinado que con la doxazosina o el finasteride. Los pacientes en tratamiento combinado y en menor medida con finasteride tuvieron una reducción significativa de riesgo de recibir procedimientos quirúrgicos a los 4 años. Ver mayores detalles en la Tabla. Las tres ramas mejoraron el flujo urinario: el promedio de aumento fue de $5.1 \mathrm{ml} / \mathrm{s}$ en la rama combinación, $4 \mathrm{ml} / \mathrm{s}$ con doxazosina, y $3.2 \mathrm{ml} / \mathrm{s}$ con finasteride. Los efectos adversos fueron más frecuentes que en la rama placebo. Doxazosina: mareos $(4 \%)$, hipotensión postural $(4 \%)$, astenia $(5 \%)$, reacciones alérgicas $(1 \%)$ y somnolencia (1\%). Rama combinación: disfunción eréctil (5\%), disminución de la libido $(3 \%)$, eyaculación anormal $(3 \%)$. Finasteride: Disfunción eréctil $(5 \%)$, disminución de la libido $(2 \%)$ y eyaculación anormal $(2 \%)$.

Tabla: resultados principales de las tres ramas activas (comparadas con la rama

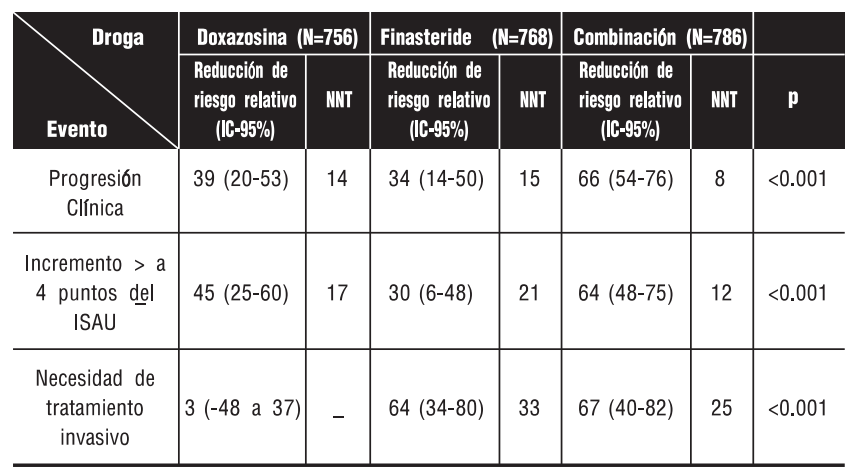

${ }^{*}$ NNT (Número necesario a tratar) calculado en base a la incidencia a los 4 años.placebo)

\section{Conclusiones}

La combinación a largo plazo de doxazosina y finasteride fue segura, y redujo el riesgo de progresión clínica de la enfermedad en mayor medida que cada una de ellas en forma aislada. El tratamiento combinado y el finasteride redujeron el riesgo a largo plazo de retención urinaria aguda y la necesidad de tratamientos invasivos.

Fuente de Financiamiento: National Institutes of Health, EE.UU, Merk y Pfizer.

\section{Comentario}

Ya está ampliamente demostrado que tanto el finasteride como la doxazosina son eficaces para reducir el riesgo de progresión de la HPB. ${ }^{1}$ También se había demostrado la eficacia de la combinación de ambas drogas en estudios a mediano y corto plazo, pero hasta el año de seguimiento no había demostrado mejores resultados que la monoterapia. ${ }^{2}$ Este estudio, realizado con una muy buena metodología, no solo extiende los resultados a largo plazo, sino que demuestra que la combinación tiene un eficacia aún mayor en la reducción de progresión de síntomas que cada droga en forma independiente. También se demuestra que con e tratamiento combinado reduce más el volumen prostático (especialmente en pacientes con próstatas de mayor tamaño), el riesgo de recibir tratamiento quirúrgico y la retención urinaria (esto último sucede probablemente a expensas del finasteride, ya que este efecto también se observó con esta droga en forma aislada). El finasteride parece ser más beneficioso en las próstatas más voluminosas y con APE $>2,25$.

\section{Conclusiones del comentador}

Este estudio llevado a la práctica, sugiere que la combinación de finasteride y doxazosina podría ser una opción en pacientes aún sintomáticos con monoterapia, antes de plantearse un tratamiento invasivo. A pesar de haber sido bien toleradas, debemos tener en cuenta que los efectos indeseables aumentan al combinarlas.

\section{Dr. Pablo Tesolin Unidad de Medicina Familiar [ Hospital Italiano de Buenos Aires ]}

Tesolin P. La combinación de finasteride y doxazosina fue más efectiva que la monoterapia en la hiperplasia prostática benigna. Evid. actual. práct. ambul. 2004;7:104-105. Comentado de: McConnell JD, Roehrborn CG, Bautista OM et al. The long-term effect of Doxazosin, Finasteride, and combination therapy on the Cinical Progression of Benign Prostatic Hyperplasia. N Engl J Med 2003;349:2387-2398.

\section{Referencias}

1 AUA practice Guidelines Committee.AUA guideline on management of benign prostatic hyperplasia (2003) J Urol 2003; $170 ; 530-47$.

2. Lepor H., Williford WO, Barry MJ y cols. The Efficacy of Terazosin, Finasteride, or Both in Benign Prostatic Hyperplasia. N Engl J Med 1996; 335:533-540 


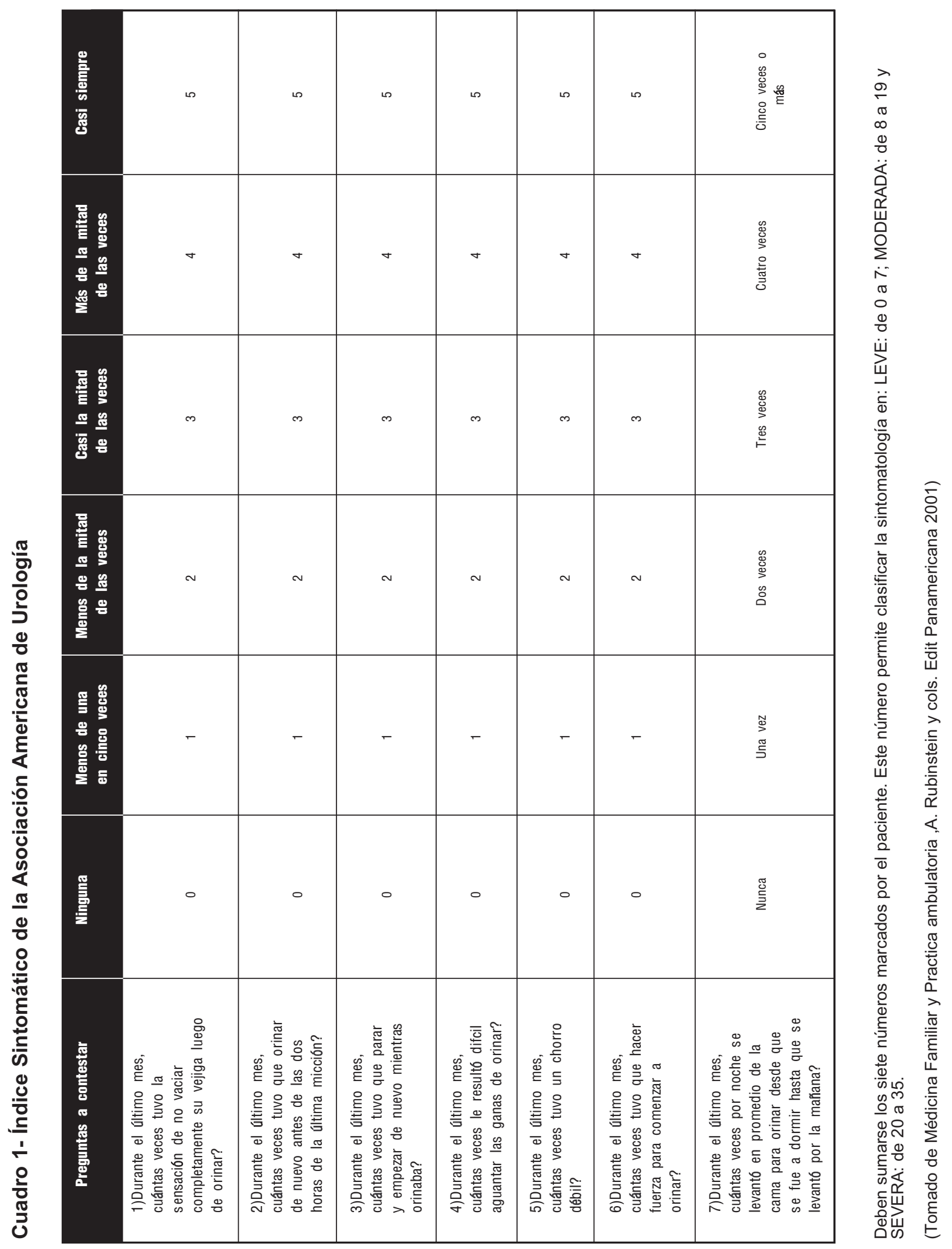

